\title{
Intrinsic Contribution of Perforin to NK-Cell Homeostasis during Mouse Cytomegalovirus Infection
}

\author{
Maja Arapović1 ${ }^{1 \dagger}$, Ilija Brizić ${ }^{2 \dagger}$, Branka Popović ${ }^{1}$, Slaven Jurković ${ }^{3}$, Stefan Jordan ${ }^{4}$, \\ Astrid Krmpotić ${ }^{1}$, Jurica Arapović ${ }^{1,5}$ and Stipan Jonjić ${ }^{1,2 *}$ \\ ${ }^{1}$ Department of Histology and Embryology, Faculty of Medicine, University of Rijeka, Rijeka, Croatia, ${ }^{2}$ Center for Proteomics, \\ Faculty of Medicine, University of Rijeka, Rijeka, Croatia, ${ }^{3}$ Department of Medical Physics, Clinical Hospital Rijeka, Rijeka, \\ Croatia, ${ }^{4}$ Department of Oncological Sciences, Tisch Cancer Institute and the Immunology Institute, Icahn School of \\ Medicine at Mount Sinai, New York, NY, USA, ${ }^{5}$ Faculty of Medicine, University of Mostar, Mostar, Bosnia and Herzegovina
}

\section{OPEN ACCESS}

Edited by:

Chiara Romagnani,

Deutsches Rheuma

Forschungszentrum, Germany

Reviewed by:

Nadia Guerra

Imperial College London, UK Vincent Vieillard,

Centre National de la Recherche

Scientifique (CNRS), France

${ }^{*}$ Correspondence:

Stipan Joniic

stipan.jonjic@medri.uniri.hr

†Maja Arapović and llija Brizić contributed equally to the work.

Specialty section:

This article was submitted to NK Cell Biology,

a section of the journal

Frontiers in Immunology

Received: 01 February 2016

Accepted: 24 March 2016

Published: 06 April 2016

Citation:

ArapovićM, Brizićl, Popović B, JurkovićS, Jordan S, Krmpotić A,

Arapović J and Jonjić S (2016) Intrinsic Contribution of Perforin to NK-Cell Homeostasis during Mouse

Cytomegalovirus Infection.

Front. Immunol. 7:133.

doi: 10.3389/fimmu.2016.00133
In addition to their role as effector cells in virus control, natural killer (NK) cells have an immunoregulatory function in shaping the antiviral T-cell response. This function is further pronounced in perforin-deficient mice that show the enhanced NK-cell proliferation and cytokine secretion upon mouse cytomegalovirus (MCMV) infection. Here, we confirmed that stronger activation and maturation of NK cells in perforin-deficient mice correlates with higher MCMV load. To further characterize the immunoregulatory potential of perforin, we compared the response of NK cells that express or do not express perforin using bone-marrow chimeras. Our results demonstrated that the enhanced proliferation and maturation of NK cells in MCMV-infected bone-marrow chimeras is an intrinsic property of perforin-deficient NK cells. Thus, in addition to confirming that NK-cell proliferation is virus load dependent, our data extend this notion demonstrating that perforin plays an intrinsic role as a feedback mechanism in the regulation of NK-cell proliferation during viral infections.

Keywords: mouse cytomegalovirus, perforin, NK cells, bone-marrow chimera, Ly49H, m157, proliferation

\section{INTRODUCTION}

Natural killer (NK) cells play a crucial role in the early stage of mouse cytomegalovirus (MCMV) infection; however, the contribution of NK cells varies among different mouse strains (1). In C57BL/6 mice, the activating NK-cell receptor Ly49H mediates resistance to MCMV infection, because of the specific binding of virally encoded $\mathrm{m} 157$ protein $(2,3)$. NK cells exert their function by releasing antiviral cytokines and by cytolytic mechanisms mediated by perforin and granzymes. Mice lacking the Ly $49 \mathrm{H}$ receptor fail to exert significant virus control by NK cells during the early post-infection (p.i.) days, because of the fact that MCMV expresses immune evasion mechanisms able to avoid or decrease other means of NK-cell engagement (4-6). In addition, MCMV downregulates major histocompatibility complex class I (MHC-I) molecules to avoid detection by $\mathrm{CD} 8^{+} \mathrm{T}$ cells (7), but at the same time, the virally encoded $m 04$ protein escorts sufficient MHC-I complexes to the cell surface to ligate inhibitory Ly 49 receptors and avoid NK-cell recognition (8). Thus, one can generalize that NK cells play a dominant role in MCMV control only in mice expressing Ly49H receptor and deletion of either the $m 157$ gene or blocking of the Ly49H receptor abolish the control in most of the organs (9-12). Perhaps the best evidence for the role of Ly49H/m157 interaction in MCMV control 
by NK cells is illustrated by strong selection pressure imposed by NK cells, resulting in numerous mutations and deletions in the $m 157$ gene after passing the virus through the Ly49 $\mathrm{H}^{+}$host (13).

Our research group along with other research groups has previously shown that NK-cell response to MCMV modulates subsequent $\mathrm{CD}^{+} \mathrm{T}$-cell response and that both specific activation of $\mathrm{NK}$ cells and perforin-dependent mechanisms are involved (14-18). In C57BL/6 mice infected with wild-type (WT) MCMV, CD8 ${ }^{+} \mathrm{T}$-cell response was markedly weaker compared to mice infected with the virus lacking $m 157$ gene, suggesting that specific virus control via Ly49H ligation dampens CD8 ${ }^{+}$T-cell response (19). It was previously shown that Ly49H ligation via $m 157$ enhances NK-cell proliferation (20). Perforin deficiency in NK cells compromises MCMV control, in spite of the fact that proliferation and production of cytokines were stronger than in WT NK cells expressing perforin (21). The immunoregulatory impact of NK cells on $\mathrm{CD}^{+} \mathrm{T}$ cells was still evident in perforindeficient C57BL/6 (Prf1 $\left.{ }^{--}\right)$mice. Under these conditions, perforin-deficient NK cells regulate $\mathrm{CD} 8^{+} \mathrm{T}$-cell response mostly by secreting inhibitory cytokine IL-10 (21). It remains unclear whether the enhanced proliferation of NK cells in $\mathrm{Prfl}^{-/-}$mice is caused by a high virus load or if it represents a homeostatic function of perforin.

Here, we aimed to elucidate the immunoregulatory potential of perforin with the emphasis on NK-cell proliferation and differentiation during infection. For the same, we used a model of bone-marrow chimeras possessing NK cells with or without perforin and tested their response to MCMV. We found that in addition to virus load-dependent $\mathrm{Ly} 49 \mathrm{H}^{+} \mathrm{NK}$-cell proliferation, perforin has an intrinsic role as a feedback mechanism in the regulation of NK-cell homeostasis during viral infections.

\section{RESULTS}

\section{Perforin Deficiency Enhances IFN- $\gamma$ Secretion and Proliferation of NK Cells during Early MCMV Infection}

To assess the impact of perforin on NK-cell response to MCMV, C57BL/ 6 and $\mathrm{Prf1}^{-1-}$ mice were infected with either WT MCMV or the virus mutant lacking $m 157(\Delta m 157)$, and virus titer was determined 3 days p.i. (Figure 1A). The proportion of mice in each group was subjected to depletion of NK cells by anti-NK1.1 monoclonal antibodies (mAbs). As shown previously $(11,22)$, MCMV control in C57BL/6 mice was almost completely dependent on Ly49H/m157 interaction. However, in perforin-deficient mice, the virus control by NK cells was essentially abolished, and no difference between WT virus and $\Delta m 157$ mutant was found in spite of the fact that significantly more NK cells in perforindeficient mice perforin produced IFN- $\gamma$, in comparison with WT control mice (Figure 1B). In C57BL/6 mice infected with $\Delta m 157$ virus, we also found higher frequency of IFN- $\gamma$-producing NK cells in comparison with WT MCMV-infected mice, which correlates with a higher virus load and higher level of IFN- $\alpha$ and IL-12 in sera of $\mathrm{Prf1}^{-/-}$mice (Figure 1C). However, in Prf1 ${ }^{-/-}$mice, higher level of cytokine production was observed irrespective of the virus used. Importantly, our results suggest that enhanced proliferation of NK cells in the absence of perforin is also driven by specific ligation of the NK-cell receptor $\mathrm{Ly} 49 \mathrm{H}$, because $\mathrm{Ly}_{4} 9 \mathrm{H}^{+}$ cells proliferate much more strongly in mice infected with WT virus, as compared with virus lacking $m 157$ [Figure 1D; $(20,21)$ ].

It is well established that MCMV infection drives maturation of NK cells toward a terminally differentiated phenotype (23). NK cells derived from MCMV-infected $\mathrm{Prf1}^{-/-}$mice behaved in a similar fashion; however, the maturation was even more enhanced than in WT mice (Figure 2A). On day 6 p.i., the vast majority of NK cells in Prf1 ${ }^{-1-}$ mice were of terminally differentiated $\mathrm{CD} 27^{-} \mathrm{CD} 11 \mathrm{~b}^{+}$phenotype. This pattern was observed even in mice infected with $\Delta m 157$ virus, although our data indicate that Ly $49 \mathrm{H} / \mathrm{m} 157$ interaction further enhanced maturation of NK cells (Figure 2B). Further evidence for terminal differentiation of NK cells in the absence of perforin was provided by following the expression of KLRG1 at different time points after infection. As shown in Figure 2C, almost all NK cells in $\mathrm{Prf1}^{-1-}$ mice expressed KLRG1 on day 6 p.i., once again confirming acquisition of a completely mature phenotype (24).

In total, here, we showed that in the absence of perforin, NK cells proliferate far more and differentiate faster. In addition, a higher frequency of NK cells secrets IFN- $\gamma$, as compared to NK cells derived from control mice. It remains unclear whether these phenotypes of NK cells reflect the inability of $\mathrm{Prfl}^{-/-}$mice to control the virus via NK cells, or if perforin plays an additional, so far uncharacterized, regulatory function.

\section{Enhanced Accumulation of Prf1-/- NK Cells Is Their Intrinsic Function}

Although the results described above clearly demonstrated the different functional properties of NK cells in the absence of perforin, we were not able to confirm that this is indeed the intrinsic effect of perforin on NK cells. This is because all these phenotypes (enhanced proliferation, faster maturation, and increased production of cytokines) could simply be a consequence of higher virus load in $\mathrm{Prf1}^{-1-}$ mice as compared to control C57BL/6 mice. To compare the proliferation and differentiation of $\mathrm{Prfl}^{-/-}$and WT NK cells in the presence of identical virus loads, we generated bone-marrow chimeric mice (Figure 3A). Bone-marrow cells derived from C57BL/6 mice $\left(\mathrm{CD} 45.1^{+}\right)$and $\mathrm{Prf1}^{-/-}$mice $\left(\mathrm{CD} 45.2^{+}\right)$were transferred at equal ratio into $\gamma$-irradiated C57BL/6 (CD45.1 $\left.1^{+} \mathrm{CD} 45.2^{+}\right)$recipient mice. Eight weeks later, chimerism was confirmed (Figure 3B), and mice were infected with either WT, $\Delta m 157$ MCMV, or left uninfected. Seven days later, mice were euthanized, and splenic and liver lymphocytes were analyzed (Figures 3C,D). In control uninfected chimeric mice, the frequency of Prf1 ${ }^{-1-} \mathrm{NK}$ cells was similar to WT NK cells, indicating no apparent impact of perforin on the generation of NK cells in steady-state conditions. However, in agreement with the results shown in Figure 1, a much higher frequency of $\mathrm{Prf1}^{-/-} \mathrm{NK}$ cells was found both in spleen and liver in WT MCMV-infected chimeric mice, as compared to WT NK cells (Figure 3C). Although the absence of $\mathrm{Ly} 49 \mathrm{H} / \mathrm{m} 157$ interaction resulted in a dramatic drop of NK-cell frequency, some differences between $\mathrm{Prf1}^{-/-}$and WT NK cells were preserved. Lower frequency of splenic NK cells and decreased differences between 
A

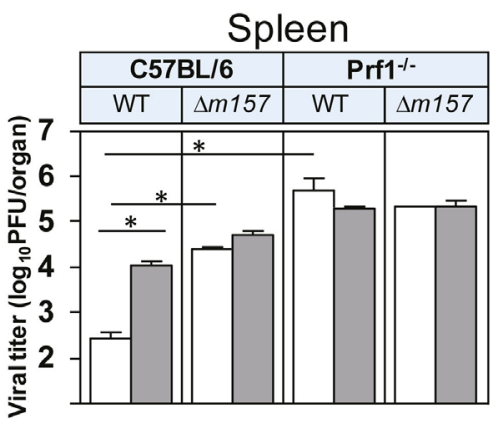

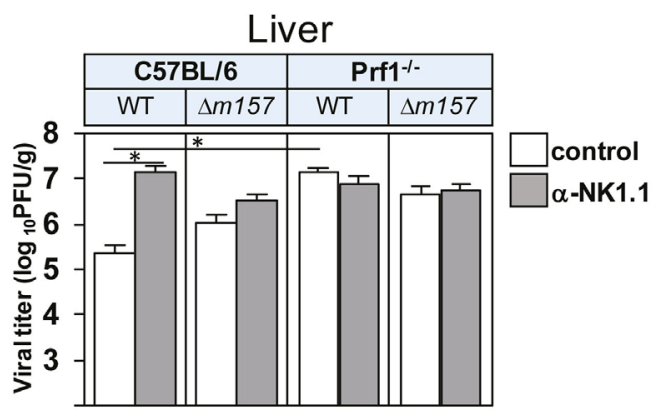

B

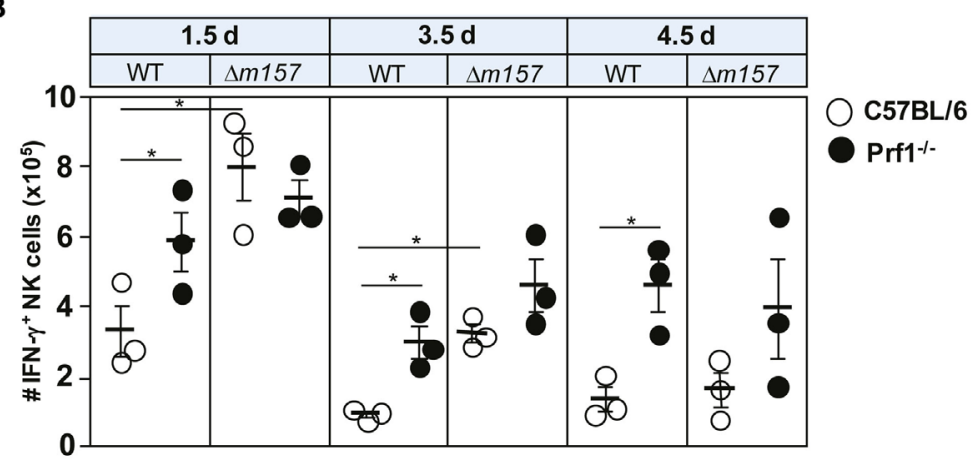

C
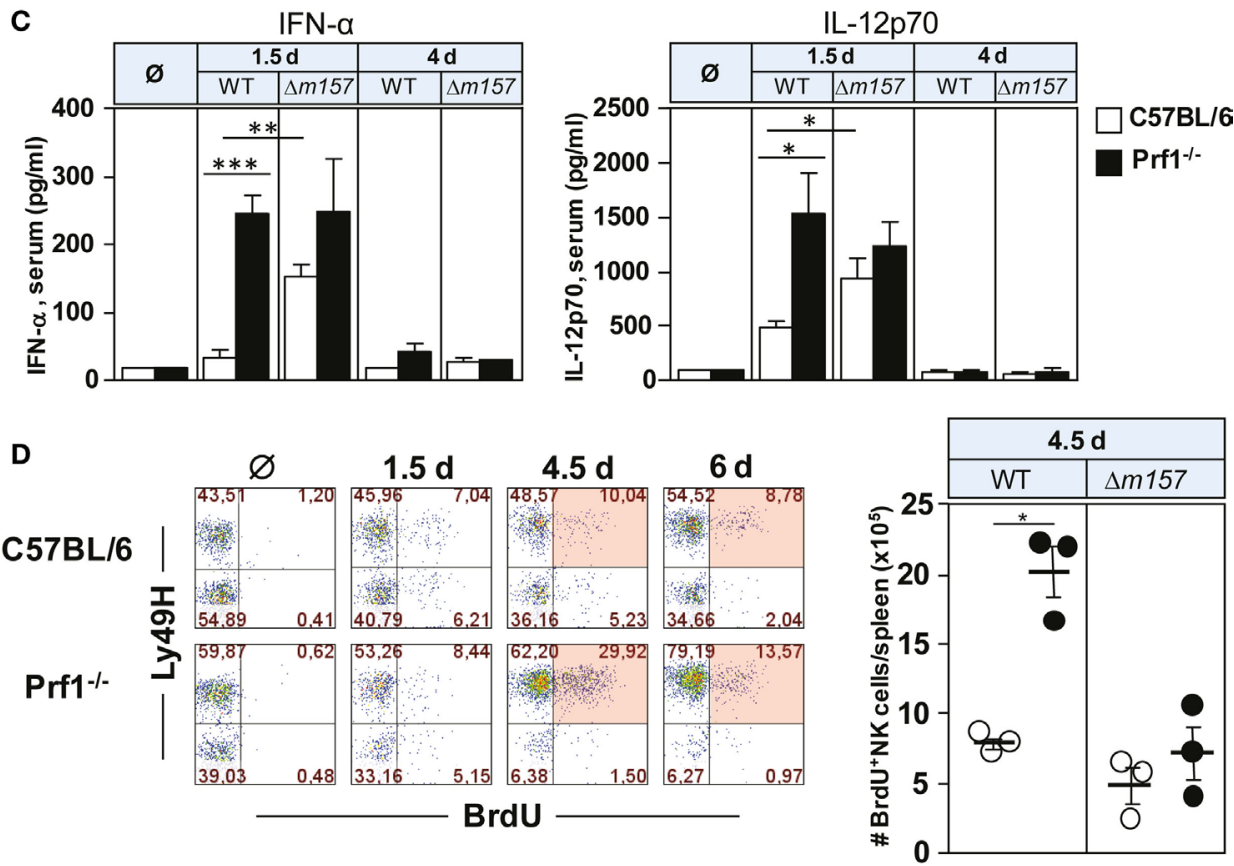

FIGURE 1 | Perforin deficiency enhances IFN- $\boldsymbol{\gamma}$ secretion and proliferation of NK cells during early MCMV infection. C57BL/6 and Prf1-/- mice were infected intravenously with $2 \times 10^{5}$ PFU of indicated viruses. (A) Mice, either NK-cell depleted or NK-cell undepleted before infection, were euthanized 3 days p.i. and titers in spleen (per organ) and liver (per gram) were determined. (B) On days 1.5, 3.5, and 4.5 p.i., splenocytes were assessed for IFN- $\gamma$ production by CD3e-NK1.1+ NK cells. (C) On days 1.5 and 4 p.i., serum levels of the indicated cytokines were determined. (D) On days 1.5, 4.5, and 6 p.i., mice were i.p. injected

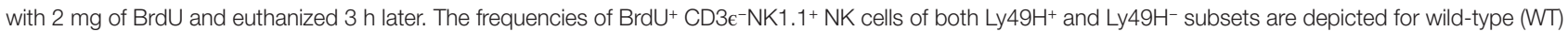
MCMV infection (left). The number of BrdU + CD3e-NK1.1+ NK cells on day 4.5 p.i. following WT and $\Delta m 157$ MCMV infection is shown (right). Representative data of at least two independent experiments with three to five mice per group are shown. Data are presented as means \pm SEM. Asterisks denote significant values: ${ }^{\star} P<0.05 ;{ }^{* *} P<0.01 ;{ }^{* *} P<0.001$. 

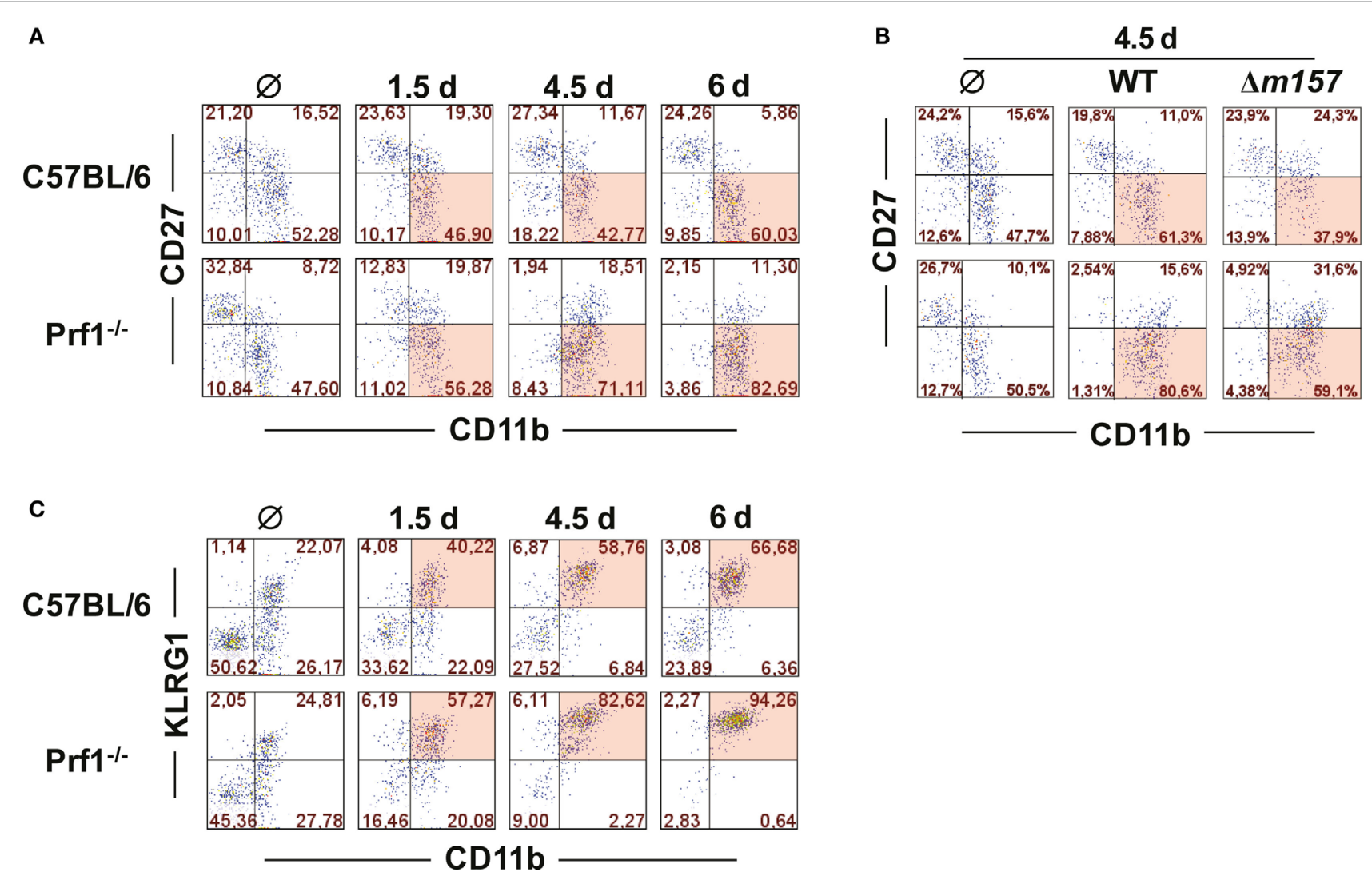

FIGURE 2 | Perforin deficiency enhances maturation of NK cells during early MCMV infection. C57BL/6 and Prf1 ${ }^{-/-}$mice were infected intraperitoneally with $10^{6} \mathrm{PFU}$ of MCMV (A,C) or intravenously with $2 \times 10^{5} \mathrm{PFU}$ of MCMV (B). (A) At indicated time points, splenic CD3e-NK1.1 $1^{+}$NK cells were analyzed for expression of CD27 and CD11b markers. (B) On day 4.5, maturation pattern of NK cells was determined for WT and $\triangle m 157$ MCMV infection. (C) Frequencies of KLRG1 and CD11b coexpression on CD3e-NK1.1+ NK cells at indicated time points p.i. are depicted. Representative data of at least two independent experiments with three to five mice per group are shown.

perforin-deficient and perforin-sufficient NKcells in mice infected with $\Delta m 157$ MCMV are likely a consequence of high virus load, as shown previously $(19,21)$. In addition, the results suggest that enhanced proliferation of perforin-deficient NK cells requires their stimulation via a specific receptor. Notably, no significant differences in frequency of $\mathrm{Prf1}^{-/-}$and $\mathrm{WT} \mathrm{CD} 8^{+} \mathrm{T}$ cells were found (Figure 3D). A slightly lower frequency of $\mathrm{CD} 8^{+} \mathrm{T}$ cells in $\mathrm{Prf1}^{-/-}$mice was observed, regardless of infection. Thus, we demonstrated that, in chimeric mice, the enhanced accumulation and differentiation of $\mathrm{Prf1}^{-1-} \mathrm{NK}$ cells is their intrinsic function, which becomes apparent during virus infection.

\section{Intrinsic Function of Perforin on NK-Cell Proliferation and Maturation Is Ly49H Dependent}

In Figure 1, we have shown that, during MCMV infection, NK cells of $\mathrm{Prf1}^{-/-}$mice proliferated more strongly, allowing them to reach the terminally differentiated phenotype faster than NK cells from control C57BL/6 mice. To rule out the impact of a different virus load, next we tested maturation pattern of NK cells in bone-marrow chimeras (Figure 4A). Here again, we observed that the frequency of terminally differentiated perforin-deficient NK cells was higher than the frequency of terminally differentiated WT NK cells. Moreover, the results also showed that the Ly $49 \mathrm{H} / \mathrm{m} 157$ interaction does play a role, because the differences between $\mathrm{Prf1}^{-1-}$ and WT NK cells were not significant in mice infected with $\Delta m 157$ virus. In line with this, the percentage of $\mathrm{Ly}_{4} 4 \mathrm{H}^{+} \mathrm{Prf1}^{-1-} \mathrm{NK}$ cells was higher in comparison with $\mathrm{Ly}_{49 \mathrm{H}^{+}}$WT NK cells (Figure 4B). Thus, the intrinsic function of perforin on NK-cell proliferation and differentiation is more evident if NK-cell activation is driven through ligation of specific receptor.

What could be the mechanism by which perforin affects proliferation and maturation of NK cells? Higher frequency of $\mathrm{Prf1}^{-1-} \mathrm{NK}$ cells in MCMV-infected mice could be either the consequence of changes in their transcriptome, enhanced proliferation (as indicated in Figure 1C), or prolonged survival. In bone-marrow chimeras, no differences in the expression of two key transcriptional factors regulating NK-cell development and differentiation, Eomes and T-bet, were detected between Prf1 $^{-1-}$ and WT NK cells (Figures 4C,D). Next, we tested the NK-cell expression of Ki-67, a marker of-cell proliferation, and $\mathrm{Bcl}-2$, an anti-apoptotic protein. As shown in Figure 4E, Ki-67 

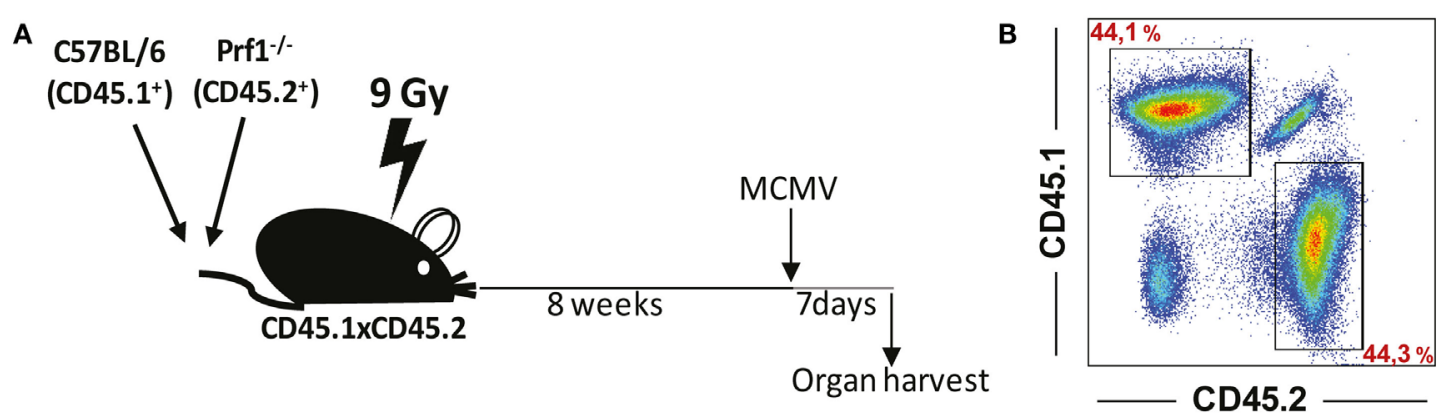

C
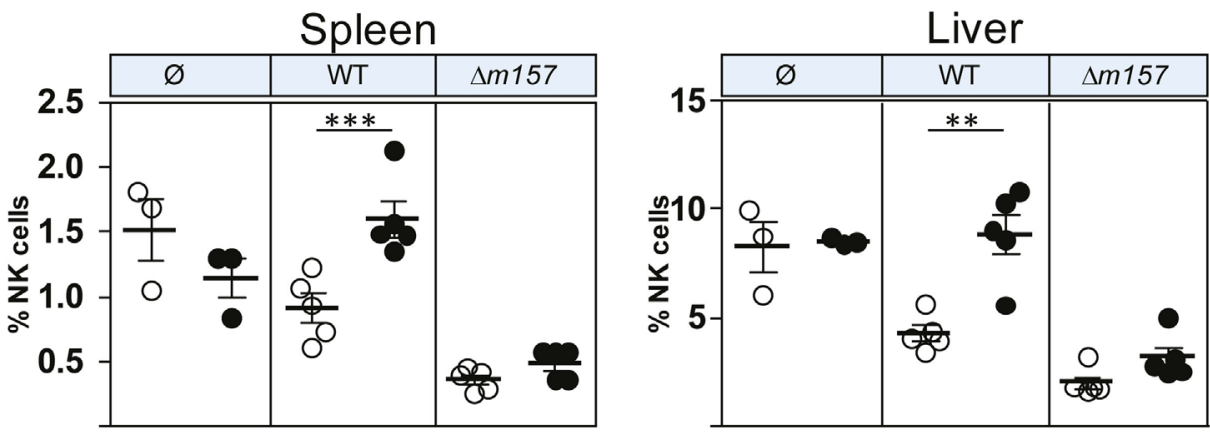

D

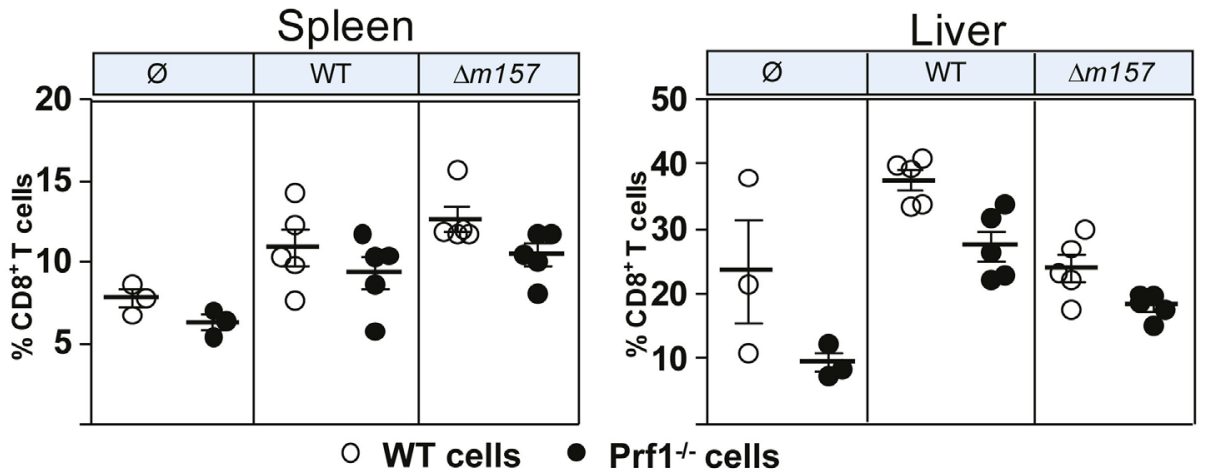

FIGURE 3 | Enhanced accumulation of perforin-deficient NK cells following MCMV infection in Prf1 ${ }^{-/-} / \mathrm{C} 57 \mathrm{BL} / 6$ chimeric mice. (A) Mixed bone-marrow chimeric mice were prepared by transferring a 1:1 mixture of C57BL/6 $\left(\mathrm{CD} 45.1^{+}\right)$and Prf1 ${ }^{-/-}\left(\mathrm{CD} 45.2^{+}\right)$bone-marrow cells to $\gamma$-irradiated CD45.1 $\times$ CD45.2 mice. Chimeric mice were i.p. injected with $5 \times 10^{5} \mathrm{PFU}$ of WT or $\triangle m 157 \mathrm{MCMV}$ and euthanized 7 days p.i. (B) Representative dot plot showing reconstitution efficiency of bone-marrow chimera. (C) Frequency of NK cells and (D) CD8 ${ }^{+}$T cells in spleen (left) and liver (right) of Prf1 $1-/ C 57 B L / 6$ chimeric mice is shown. Representative data of two independent experiments with three to five mice per group are shown. Data are presented as means \pm SEM. Asterisks denote significant values: ${ }^{\star} P<0.05 ;{ }^{* \star} P<0.01 ;{ }^{\star \star \star} P<0.001$.

was expressed in a greater proportion of $\mathrm{Prf1}^{-1-} \mathrm{NK}$ cells than in WT NK cells. This was the case even in mice infected with the virus lacking $m 157$ gene. In contrast to Ki-67, expression of Bcl-2 appears to be lower in $\mathrm{Prf1}^{-/-} \mathrm{NK}$ cells (Figure 4F).

Our next goal was to confirm that Prf1 ${ }^{-/-} \mathrm{NK}$ cells indeed have a stronger capacity to proliferate compared to WT NK cells. To that aim, we used the adoptive transfer model in which we transferred CFSE-labeled Prf1 ${ }^{-/-}\left(\mathrm{CD} 45.2^{+}\right)$and WT $\left(\mathrm{CD} 45.1^{+}\right)$splenocytes into MCMV-infected CD45.1 $1^{+} \mathrm{CD} 45.2^{+}$ recipients (Figure 5A). Mice were euthanized on day 5 p.i., and CFSE dilution in NK cells was analyzed. In line with our data obtained in bone-marrow chimeras, we observed higher proportion of donor derived $\mathrm{Prf1}^{-/-} \mathrm{NK}$ cells compared to
$\mathrm{Prf1}^{+/+} \mathrm{NK}$ cells following adoptive transfer (Figure 5B). In agreement with Ki-67 expression, CFSE was more diluted in $\mathrm{Prf1}^{-/-} \mathrm{NK}$ cells than in Prf1 ${ }^{+/+} \mathrm{NK}$ cells (Figure 5C). Thus, our results confirmed that, in addition to serving as a major cytolytic molecule, perforin also plays an important intrinsic role by influencing proliferation capacity and differentiation of NK cells during virus infection.

\section{DISCUSSION}

Here, we describe a novel function of perforin in the regulation of NK-cell proliferation and differentiation during MCMV infection. In agreement with the previously described studies, 
A

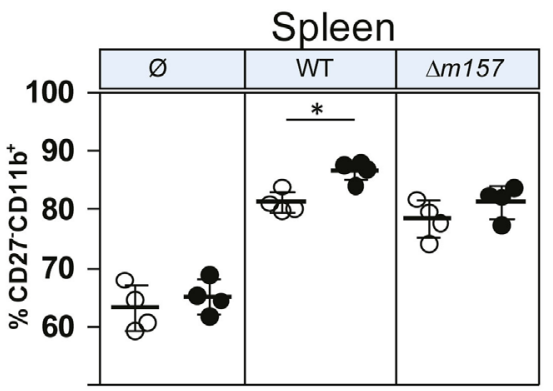

B

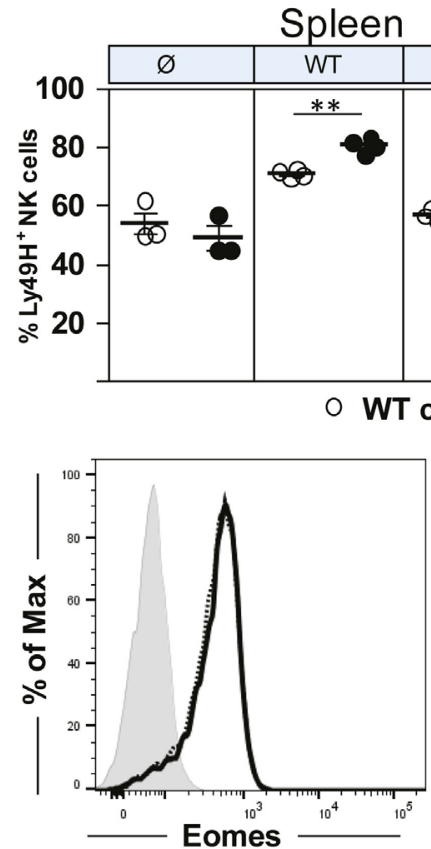

D

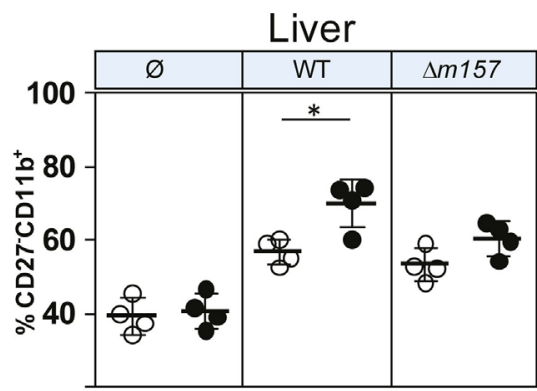

Liver

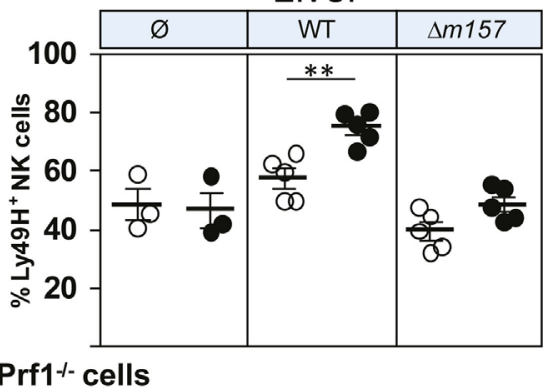

C
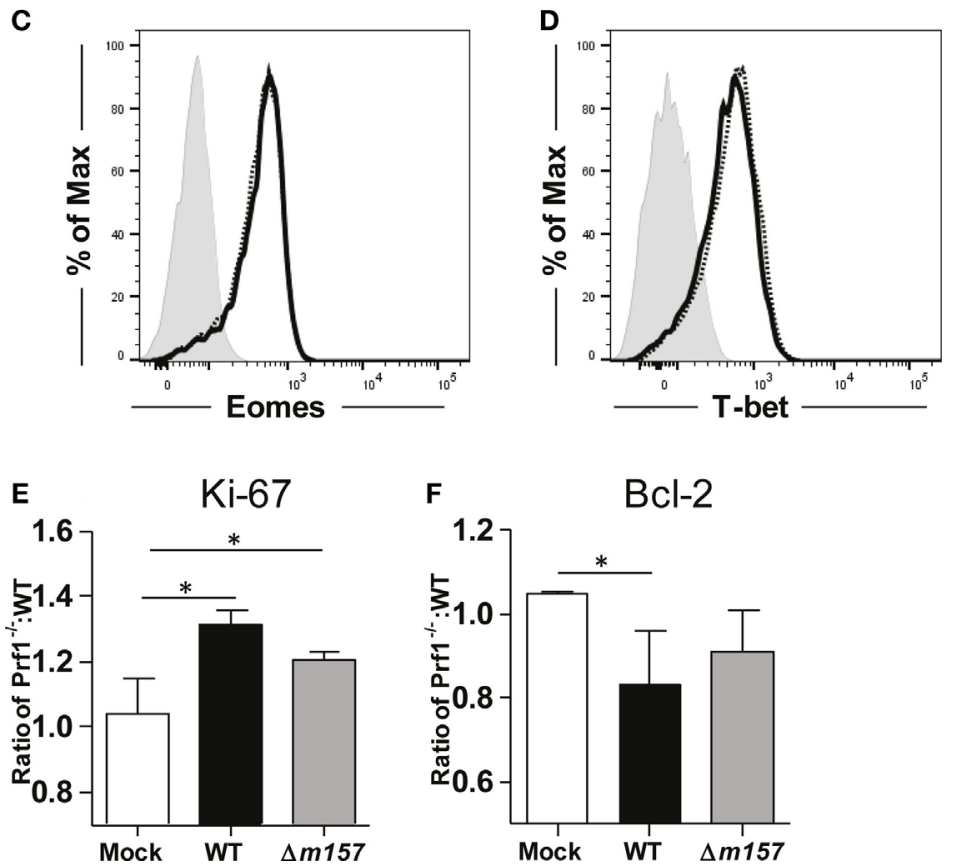

FIGURE 4 | NK-cell phenotype in Prf1 ${ }^{-l-} /$ C57BL/6 BM chimeric mice. (A) Percentage of terminally differentiated CD27-CD11 b+ and (B) Ly49H+ $\mathrm{NK}^{+}$cells is shown in spleen (left) and liver (right) of WT MCMV, $\triangle m 157$ MCMV, and mock-infected Prf1-/-/C57BL/6 BM chimeric mice. Representative histograms of (C) Eomes and (D) T-bet are shown. Relative expression of (E) Ki-67 and (F) Bcl-2 is shown calculated as ratio of expression of each marker in Prf1 $1^{-/-} \mathrm{NK}$ cells and C57BL/6 NK cells. Representative data of two independent experiments with three to five mice per group are shown. Data are presented as means \pm SEM. Asterisks denote significant values: ${ }^{*} P<0.05 ;{ }^{* \star} P<0.01 ;{ }^{* \star *} P<0.001$.

we first confirmed that in the absence of perforin, NK cells proliferate more strongly upon infection, compared to NK cells from control mice expressing this cytolytic molecule. Furthermore, this enhanced proliferation is accompanied with increased IFN- $\gamma$ secretion and NK-cell maturation. The enhanced proliferation is much more evident if activating
NK-cell receptor Ly49H is engaged. Yet, it remains unclear whether this is only a consequence of higher virus load, because the virus control is almost completely compromised, and infection is accompanied by high level of proinflammatory cytokines in the absence of perforin. To provide equal conditions for both perforin-deficient and perforin-sufficient 

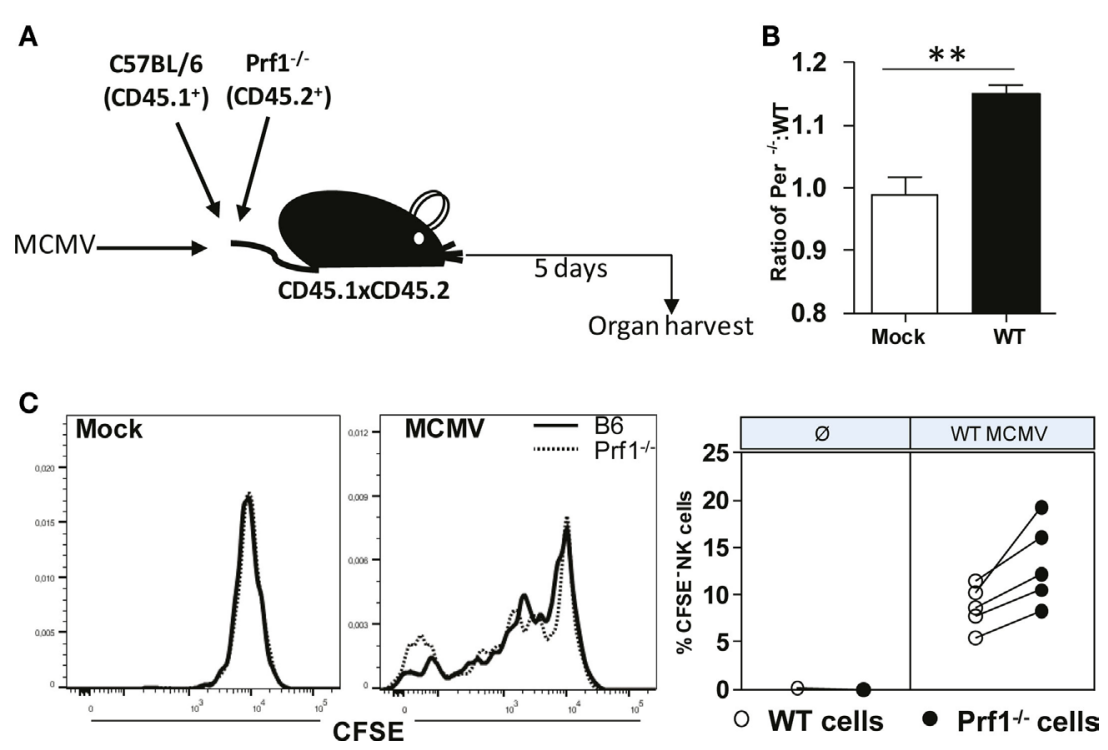

FIGURE 5 | Enhanced dilution of CFSE by perforin-deficient NK cells following MCMV infection in Prf1 ${ }^{-/-} / \mathrm{C} 57 \mathrm{BL} / 6$ chimeric mice. (A) A 1:1 mixture of C57BL/6 $\left(\mathrm{CD} 45.1^{+}\right)$and Prf1-/- $\left(\mathrm{CD} 45.2^{+}\right)$CSFE-labeled splenocytes were adoptively transferred to CD45.1 $\times$CD45.2 mice that were infected with MCMV $3 \mathrm{~h}$ prior to transfer. (B) Ratio of number of adoptively transferred Prf1 ${ }^{-/-}$and C57BL/6 NK cells is depicted. (C) Proliferation of adoptively transferred Prf1 ${ }^{-/-}$and C57BL/6 NK cells in spleen was determined by analyzing CFSE dilution on day 5 p.i. Representative histograms for mock and MCMV-infected mice (left), and quantification of CFSE- NK cells (right) are shown. Asterisks denote significant values: ${ }^{*} P<0.05 ;{ }^{* \star} P<0.01 ;{ }^{* \star} P<0.001$.

NK cells, we used bone-marrow chimeras and demonstrated that perforin-deficient NK cells still proliferate more and differentiate faster, confirming the intrinsic function of perforin in NK-cell response to viral infections. Note that these differences were absent in uninfected animals, confirming that perforin deficiency does not compromise NK-cell maturation and differentiation under normal conditions.

At first glance, our results are in disagreement with the results published by Lee et al., who showed that coculture of NK cells with cells expressing $m 157$ gene results in equal proliferation of perforin-deficient and perforin-sufficient NK cells (21). However, these two models are not fully compatible, because in vivo infection conditions provide a different microenvironment, which is apparently essential for the phenotype that we observed. Several mechanisms could explain higher levels of perforin-deficient NK cells: (i) higher virus load and stronger cytokine response, (ii) homeostatic elimination by cytolytic cells, and (iii) prolonged interaction and enhanced activation of NK cells via the intercellular synapses with the infected cells. Lee et al. showed that an increase in virus load correlates with enhanced proliferation of NK cells (21). However, in bone-marrow chimeras, perforindeficient and perforin-sufficient NK cells are exposed to the same virus load and cytokine environment. Another way by which perforin deficiency could result in enhanced accumulation of NK cells could be as a consequence of decreased elimination of these cells by perforin-expressing effector cells. It is well established that perforin regulates immune cell homeostasis. During LCMV infection, perforin-mediated killing is involved in deletion of anergic antigen-specific $\mathrm{CD}^{+} \mathrm{T}$ cells (25). Furthermore, regulatory T cells can induce death of tumor infiltrating $\mathrm{NK}$ and $\mathrm{CD}^{+} \mathrm{T}$ cells in a perforin-dependent manner (26). NK cells can also eliminate other cells as a part of their homeostatic function. Crouse et al. have previously shown that, in mice lacking type I IFN receptor, $\mathrm{CD}^{+} \mathrm{T}$ cells are highly susceptible to NK-cell killing in a perforindependent manner (27). NK cells can also commit fratricide in certain conditions and by doing so participate in NK-cell homeostasis $(28,29)$. However, the differences in accumulation of NK cells between $\mathrm{Prfl}^{-/-}$and WT mice should be abolished in chimeric mice. Although, in this work, we were unable to characterize the ultimate mechanisms by which perforin regulates NK-cell maturation, it is worth mentioning that the recent study by Jenkins et al. showing that perforin-deficient NK cells form prolonged synapses with target cells (30). This leads to repetitive calcium signaling and enhanced production of cytokines. Interestingly, perforin-deficient NK cells remained in synapse with their targets for a significantly longer period of time than granzyme A- and B-deficient NK cells, but there was no difference in the case of $\mathrm{CD}^{+} \mathrm{T}$ cells lacking these cytolytic molecules. In addition, as proposed by Sad et al., prolonged interaction because of inability of cytotoxic cells to kill target cells may be sufficient for sustained stimulation and activation of effector cells (31). It needs to be tested whether or not this could explain better survival and enhanced differentiation of perforin-deficient NK cells.

Negative feedback mechanisms are essential for any physiological function. Here, we described the novel regulatory function of perforin in homeostasis of NK cells during virus infection, and excluded several mechanisms that have been described to be involved in homeostatic regulation of NK cells and other effector cells. Further studies are required to fully elucidate this novel immunoregulatory role of perforin during viral infection. 


\section{MATERIALS AND METHODS}

\section{Mice}

$\mathrm{C} 57 \mathrm{BL} / 6, \mathrm{Prf1}^{-/-}$(32), $\mathrm{C} 57 \mathrm{BL} / 6 \mathrm{CD} 45.1^{+}$, and $\mathrm{C} 57 \mathrm{BL} / 6$ $\mathrm{CD} 45.1^{+} \mathrm{CD} 45.2^{+}$mice were housed and bred under specificpathogen-free conditions at the Central Animal Facility of the Medical Faculty, University of Rijeka, in accordance with the guidelines contained in the International Guiding Principles for Biomedical Research Involving Animals. The Animal Welfare Committee at the University of Rijeka approved all animal experiments described in this paper. Eight- to 12-week-old mice were used in all experiments.

\section{Viruses}

Mice were injected intravenously (i.v.) or i.p. with $2-5 \times 10^{5} \mathrm{PFU}$ of the tissue culture (TC)-grown virus in a volume of $500 \mu \mathrm{l}$ of diluent (PBS or DMEM media). Bacterial artificial chromosome (BAC)-derived MCMV strain MW97.01 has previously been shown to be biologically equivalent to MCMV strain Smith (VR1399) and is hereafter referred to as WT MCMV (33). In addition to MW97.01, the mutant virus lacking $m 157$ gene was used (9).

\section{Depletion of Lymphocyte Subsets and the Determination of Viral Titers In Vivo}

The depletion of NK cells in vivo was performed by intraperitoneal injection of $300 \mu \mathrm{g}$ of purified MAb PK136 (34) at 1 day before infection and 1 day p.i. For quantifying viral infectivity in organs, virus titers were determined by standard plaque assay, as described previously (35).

\section{Flow Cytometry and Intracellular Staining}

Splenic leukocytes were prepared, as previously described, and in order to decrease non-specific staining, Fc receptors were blocked with $2.4 \mathrm{G} 2 \mathrm{mAbs}$ (36). The following $m A b s$ were purchased from eBioscience or BD Pharmingen, and cell-surface staining was performed specifically for the following antigens: anti-CD3e (145-2C11), anti-NK1.1 (PK136), anti-Ly49H (3D10), anti-CD27 (LG.7F9), anti-CD11b (M1/70), anti-CD8 $\alpha$ (53-6.7), anti-IFN- $\gamma$ (XMG1.2), anti-CD19 (1D3), anti-KLRG1 (2F1), anti-Ki-67 (SolA15), anti-Bcl-2 (10C4), and PE-Cyanine7-labeled streptavidin (SA-PE-Cy7). For the in vivo cell proliferation assay, mice were i.p. injected with $2 \mathrm{mg}$ of bromodeoxyuridine (BrdU; Sigma) and euthanized $3 \mathrm{~h}$ later. To detect incorporated BrdU, splenic leukocytes were first stained for surface antigens and then fixed, permeabilized, refixed, treated with DNase I, and intracellularly stained according to the manufacturer's protocol (BrdU flow kit; BD Pharmingen). For the detection of IFN- $\gamma$ expression by NK cells, incubation was performed in RPMI medium supplemented with $10 \%$ of fetal calf serum (FCS; Gibco) for $5 \mathrm{~h}$ in the presence of $500 \mathrm{IU} / \mathrm{ml}$ of interleukin-2 (IL-2) at $37^{\circ} \mathrm{C}$, with $1 \mu \mathrm{g} / \mathrm{ml}$ of brefeldin $\mathrm{A}$ (eBioscience) added for the last $4 \mathrm{~h}$ of incubation. After incubation, cells were first-surface stained and then fixed and permeabilized using Cytofix/Cytoperm solutions (BD Pharmingen) followed by intracellular IFN- $\gamma$ staining, according to the manufacturer's protocol. Staining of Ki-67 was done with the FoxP3 staining buffer set (eBioscience). For Bcl-2 intracellular staining, permeabilization and fixation of cells were done with the Intracellular Fixation and Permeabilization Buffer Set (eBioscience). Flow cytometry was performed on FACSCalibur and FASCAria (BD Bioscience; San Jose, CA, USA), and data were analyzed using the FlowJo software (Tree Star).

\section{Quantification of Serum Cytokine Levels}

Serum levels of IFN- $\alpha$ were determined by an enzyme-linked immunosorbent assay (ELISA) kit for IFN- $\alpha$ (PBL Biomedical Laboratories), according to the manufacturer's instructions. Serum levels of IL-12 were determined by Bio-Rad mouse cytokine multiplex assay, according to the manufacturer's instructions (Bio-Rad Laboratories, Hercules, CA, USA).

\section{Bone-Marrow Chimeras}

Bone-marrow chimeras were prepared using 8-week-old mice as donors and recipients. Briefly, C57BL/6 $\left(\mathrm{CD} 45.1^{+} \mathrm{CD} 45.2^{+}\right)$ recipient mice were $\gamma$-irradiated with $9 \mathrm{~Gy}$. After $24 \mathrm{~h}$, recipient mice were injected i.v. with $10^{7}$ of donor bone-marrow cells containing a 1:1 mixture of C57BL/6 (CD45.1 $\left.1^{+}\right)$and $\mathrm{Prf1}^{-{ }^{-}}\left(\mathrm{CD} 45.2^{+}\right)$ bone-marrow cells. Recipient mice were maintained on antibiotic water containing enrofloxacin for 2 weeks following irradiation. Chimerism was evaluated 7 weeks post-transfer, and chimeras were used in experiments after 8 weeks of reconstitution.

\section{Adoptive Transfer of CFSE-Labeled Splenocytes}

Splenic lymphocytes were isolated from C57BL/6 and $\mathrm{Prf1}^{-/-}$mice and mixed at a ratio of 1:1. Splenocyte mixture was labeled with CFSE, as described previously (37). Briefly, cells were washed twice with PBS and suspended in 5- $\mu \mathrm{M}$ CFSE solution, followed by $12 \mathrm{~min}$ of incubation at $37^{\circ} \mathrm{C}$. The cells were washed three times in complete RPMI media supplemented with 5\% FCS and resuspended in complete RPMI. To verify the CFSE labeling of cells, samples were analyzed by flow cytometry. Recipient, nonirradiated C57BL $/ 6 \mathrm{CD} 45.1^{+} \mathrm{CD} 45.2^{+}$mice were injected i.v. with $5 \times 10^{7}$ of CFSE-labeled cells.

\section{Statistical Analysis}

Statistical analysis was carried out using Prism 5 (GraphPad Software, La Jolla, CA, USA). Statistically significant differences between two data sets in cytokine assays and phenotype analyses were determined by the unpaired two-tailed Student's $t$-test, and $P$ values of $<0.05$ were considered significant. Differences in viral titers were determined by two-tailed Mann-Whitney $U$-test.

\section{ETHICS STATEMENT}

The study was approved by the ethical committee of the Animal Welfare Committee at the University of Rijeka.

\section{AUTHOR CONTRIBUTIONS}

MA, IB, BP, AK, Stefan Jordan, and JA performed experiments. Slaven Jurkovic provided technical support. Stipan Jonjić, 
AK, MA, IB, BP, and JA designed experiments and wrote the manuscript.

\section{ACKNOWLEDGMENTS}

We thank Lars Dölken, Luka Čičin Šain, and Vanda Juranić Lisnić for critical readings of the manuscript.

\section{REFERENCES}

1. Scalzo AA, Yokoyama WM. CMV1 and natural killer cell responses to murine cytomegalovirus infection. Curr Top Microbiol Immunol (2008) 321:101-22. doi:10.1007/978-3-540-75203-5_5

2. Smith HR, Heusel JW, Mehta IK, Kim S, Dorner BG, Naidenko OV, et al. Recognition of a virus-encoded ligand by a natural killer cell activation receptor. Proc Natl Acad Sci U S A (2002) 99(13):8826-31. doi:10.1073/ pnas.092258599

3. Arase H, Mocarski ES, Campbell AE, Hill AB, Lanier LL. Direct recognition of cytomegalovirus by activating and inhibitory NK cell receptors. Science (2002) 296(5571):1323-6. doi:10.1126/science.1070884

4. Lanier LL. Evolutionary struggles between NK cells and viruses. Nat Rev Immunol (2008) 8(4):259-68. doi:10.1038/nri2276

5. Brizic I, Rovis TL, Krmpotic A, Jonjic S. MCMV avoidance of recognition and control by NK cells. Semin Immunopathol (2014) 36(6):641-50. doi:10.1007/ s00281-014-0441-9

6. Lisnić B, Lisnić VJ, Jonjić S. NK cell interplay with cytomegaloviruses. Curr Opin Virol (2015) 15:9-18. doi:10.1016/j.coviro.2015.07.001

7. Hengel H, Reusch U, Gutermann A, Ziegler H, Jonjic S, Lucin P, et al. Cytomegaloviral control of MHC class I function in the mouse. Immunol Rev (1999) 168:167-76. doi:10.1111/j.1600-065X.1999.tb01291.x

8. Babić M, Pyzik M, Zafirova B, Mitrović M, Butorac V, Lanier LL, et al. Cytomegalovirus immunoevasin reveals the physiological role of "missing self” recognition in natural killer cell dependent virus control in vivo. J Exp Med (2010) 207(12):2663-73. doi:10.1084/jem.20100921

9. Bubić I, Wagner M, Krmpotić A, Saulig T, Kim S, Yokoyama WM, et al. Gain of virulence caused by loss of a gene in murine cytomegalovirus. J Virol (2004) 78(14):7536-44. doi:10.1128/JVI.78.14.7536-7544.2004

10. Sumaria N, van Dommelen SL, Andoniou CE, Smyth MJ, Scalzo AA, DegliEsposti MA. The roles of interferon-gamma and perforin in antiviral immunity in mice that differ in genetically determined NK-cell-mediated antiviral activity. Immunol Cell Biol (2009) 87(7):559-66. doi:10.1038/icb.2009.41

11. Brown MG, Dokun AO, Heusel JW, Smith HR, Beckman DL, Blattenberger EA, et al. Vital involvement of a natural killer cell activation receptor in resistance to viral infection. Science (2001) 292(5518):934-7. doi:10.1126/ science. 1060042

12. Lee SH, Girard S, Macina D, Busà M, Zafer A, Belouchi A, et al. Susceptibility to mouse cytomegalovirus is associated with deletion of an activating natural killer cell receptor of the C-type lectin superfamily. Nat Genet (2001) 28(1):42-5. doi:10.1038/ng0501-42

13. French AR, Pingel JT, Wagner M, Bubic I, Yang L, Kim S, et al. Escape of mutant double-stranded DNA virus from innate immune control. Immunity (2004) 20(6):747-56. doi:10.1016/j.immuni.2004.05.006

14. Arapović J, Arapović M, Golemac M, Traven L, Tomac J, Rumora D, et al. The specific NK cell response in concert with perforin prevents CD8(+) $\mathrm{T}$ cell-mediated immunopathology after mouse cytomegalovirus infection. Med Microbiol Immunol (2015) 204(3):335-44. doi:10.1007/ s00430-015-0409-y

15. Mitrović M, Arapović J, Traven L, Krmpotić A, Jonjić S. Innate immunity regulates adaptive immune response: lessons learned from studying the interplay between NK and CD8+ T cells during MCMV infection. Med Microbiol Immunol (2012) 201(4):487-95. doi:10.1007/s00430-012-0263-0

16. Biron CA. Yet another role for natural killer cells: cytotoxicity in immune regulation and viral persistence. Proc Natl Acad Sci U S A (2012) 109(6):1814-5. doi:10.1073/pnas.1120528109

\section{FUNDING}

Stipan Jonjić is supported by the European Research Council (ERC) Advanced Grant (Grant number: 322693) and by the Helmholtz Virtual Institute "Viral strategies of immune evasion" (VH VI-424). This work has been supported in part by the Croatian Science Foundation under the project 7132 (AK).

17. Robbins SH, Bessou G, Cornillon A, Zucchini N, Rupp B, Ruzsics Z, et al Natural killer cells promote early CD8 T cell responses against cytomegalovirus. PLoS Pathog (2007) 3(8):e123. doi:10.1371/journal.ppat.0030123

18. Andrews DM, Estcourt MJ, Andoniou CE, Wikstrom ME, Khong A, Voigt $\mathrm{V}$, et al. Innate immunity defines the capacity of antiviral $\mathrm{T}$ cells to limit persistent infection. J Exp Med (2010) 207(6):1333-43. doi:10.1084/ jem.20091193

19. Mitrović M, Arapović J, Jordan S, Fodil-Cornu N, Ebert S, Vidal SM, et al. The NK cell response to mouse cytomegalovirus infection affects the level and kinetics of the early CD8(+) T-cell response. J Virol (2012) 86(4):2165-75. doi:10.1128/JVI.06042-11

20. Dokun AO, Kim S, Smith HR, Kang HS, Chu DT, Yokoyama WM. Specific and nonspecific NK cell activation during virus infection. Nat Immunol (2001) 2(10):951-6. doi:10.1038/ni714

21. Lee SH, Kim KS, Fodil-Cornu N, Vidal SM, Biron CA. Activating receptors promote NK cell expansion for maintenance, IL-10 production, and CD8 T cell regulation during viral infection. J Exp Med (2009) 206(10):2235-51. doi: $10.1084 /$ jem.20082387

22. Tay $\mathrm{CH}$, Welsh RM. Distinct organ-dependent mechanisms for the control of murine cytomegalovirus infection by natural killer cells. J Virol (1997) 71(1):267-75.

23. Robbins SH, Tessmer MS, Mikayama T, Brossay L. Expansion and contraction of the NK cell compartment in response to murine cytomegalovirus infection. J Immunol (2004) 173(1):259-66. doi:10.4049/jimmunol.173.1.259

24. Huntington ND, Tabarias H, Fairfax K, Brady J, Hayakawa Y, Degli-Esposti $\mathrm{MA}$, et al. NK cell maturation and peripheral homeostasis is associated with KLRG1 up-regulation. J Immunol (2007) 178(8):4764-70. doi:10.4049/ jimmunol.178.8.4764

25. Matloubian M, Suresh M, Glass A, Galvan M, Chow K, Whitmire JK, et al. A role for perforin in downregulating T-cell responses during chronic viral infection. J Virol (1999) 73(3):2527-36.

26. Cao X, Cai SF, Fehniger TA, Song J, Collins LI, Piwnica-Worms DR, et al. Granzyme B and perforin are important for regulatory $\mathrm{T}$ cell-mediated suppression of tumor clearance. Immunity (2007) 27(4):635-46. doi:10.1016/j. immuni.2007.08.014

27. Crouse J, Bedenikovic G, Wiesel M, Ibberson M, Xenarios I, Von Laer D, et al. Type I interferons protect T cells against NK cell attack mediated by the activating receptor NCR1. Immunity (2014) 40(6):961-73. doi:10.1016/j. immuni.2014.05.003

28. Madera S, Rapp M, Firth MA, Beilke JN, Lanier LL, Sun JC. Type I IFN promotes NK cell expansion during viral infection by protecting NK cells against fratricide. JExp Med (2016) 213(2):225-33. doi:10.1084/jem. 20150712

29. Taniguchi RT, Guzior D, Kumar V. 2B4 inhibits NK-cell fratricide. Blood (2007) 110(6):2020-3. doi:10.1182/blood-2007-02-076927

30. Jenkins MR, Rudd-Schmidt JA, Lopez JA, Ramsbottom KM, Mannering SI, Andrews DM, et al. Failed CTL/NK cell killing and cytokine hypersecretion are directly linked through prolonged synapse time. JExp Med (2015) 212(3):307-17. doi:10.1084/jem.20140964

31. Sad S, Kägi D, Mosmann TR. Perforin and Fas killing by CD8+ T cells limits their cytokine synthesis and proliferation. J Exp Med (1996) 184(4):1543-7. doi:10.1084/jem.184.4.1543

32. Kägi D, Ledermann B, Bürki K, Seiler P, Odermatt B, Olsen KJ, et al. Cytotoxicity mediated by $\mathrm{T}$ cells and natural killer cells is greatly impaired in perforin-deficient mice. Nature (1994) 369(6475):31-7. doi: $10.1038 / 369031 \mathrm{a} 0$ 
33. Wagner M, Jonjic S, Koszinowski UH, Messerle M. Systematic excision of vector sequences from the BAC-cloned herpesvirus genome during virus reconstitution. J Virol (1999) 73(8):7056-60.

34. Koo GC, Peppard JR. Establishment of monoclonal anti-Nk-1.1 antibody. Hybridoma (1984) 3(3):301-3. doi:10.1089/hyb.1984.3.301

35. Jonjic S, Krmpotic A, Arapovic J, Koszinowski UH. Dissection of the antiviral NK cell response by MCMV mutants. Methods Mol Biol (2008) 415:127-49. doi:10.1007/978-1-59745-570-1_8

36. Yokoyama WM, Kim S. Analysis of individual natural killer cell responses. Methods Mol Biol (2008) 415:179-96. doi:10.1007/978-1-59745-570-1_11

37. Lyons AB, Parish CR. Determination of lymphocyte division by flow cytometry. J Immunol Methods (1994) 171(1):131-7. doi:10.1016/0022-1759(94)90236-4
Conflict of Interest Statement: The authors declare that the research was conducted in the absence of any commercial or financial relationships that could be construed as a potential conflict of interest.

Copyright (c) 2016 Arapović, Brizić, Popović, Jurković, Jordan, Krmpotić, Arapović and Jonjić. This is an open-access article distributed under the terms of the Creative Commons Attribution License (CC BY). The use, distribution or reproduction in other forums is permitted, provided the original author(s) or licensor are credited and that the original publication in this journal is cited, in accordance with accepted academic practice. No use, distribution or reproduction is permitted which does not comply with these terms. 